\title{
The Views of Directors of Health Institutions Relating to the Current Situation of the Healthcare System in the Republic of Macedonia and Their Expectations for the Future
}

\section{Valdrina Fetai}

PhD Cand. University of Tetovo, Republic of Macedonia, University Lecturer

\section{Abstract}

Health institutions as an integral part of the healthcare system operate in a dynamic and complex environment in which they are constantly faced with numerous shocks from the economic, technical, technological, political, sociocultural and demographic changes of the uncontrolled external environment that are expected to be much more challenging in the next decade. The purpose of this paper is to identify how the top management of health care institutions assess the current state of the health system and how they prepare for the future of this system. This research is conducted through an anonymous annual survey of responsible persons of the decision-making hierarchy in the public health institutions. Directors of health institutions predict that in the future the new model of value-based healthcare will reformulate and change the future of healthcare. The technological advancement is the one that will significantly improve future healthcare by making the services more accessible, information more transparent as well as prepare the doctors and other medical staff to be able to provide a better healthcare. It can be concluded that healthcare managers feel great uncertainty about the dynamic and complex environment they are operating in, the overall economic situation and the possibility of increasing income. They are focused mainly on the implementation of contemporary technology for creating new values in new forms, developing new and dynamic partnerships and reducing the spending.

Keywords: The views of managers of health institutions; expectations for the future of healthcare system; Value-Based Healthcare Model; Orientation towards patients; the technological advancement

\section{Introduction}

The health system in general, lidership in this system and its development is increasingly attracting the attention of society. In front of the institutional leaders, there is a great challenge to understand in a transparent way the world we live in 
today and be able to contemplate the future and the possible changes in it. Considering their daily activities, the top managers are required to be more innovative, more flexible, to respect the knowledge and experience of employees at all levels of the institution, to advance teamwork, to advance communication between departments and units and to reward the employees for the quality work accomplished.

Health institutions as an integral part of the healthcare system operate in a dynamic and complex environment in which they are constantly faced with numerous shocks from the economic, technical, technological, political, socio-cultural and demographic changes of the uncontrolled external environment. The directors of the health institutions develop their managerial and economical activity in this complex environment with numerous, fast, and frequent changes that are expected to be much more challenging in the next decade.

The healthcare management model has undergone many radical changes that have significantly influenced the quality of health services and citizen behavior towards this very important segment of social life and its overall functioning. Today, the top management of health institutions composed by two-person directorate - the Medical Director and the Organizational Director present an innovation aimed at increasing managerial efficiency and increasing transparency in the management of human, financial and material resources, respectively in quality management of health institutions.

\section{The Purpose of the Study}

Is to identify the views of health care directors about the current state of the health system and their predictions, together with their plans for the future of it, respectively, how the top management of health care institutions assess the current state of the health system and how is preparing for the future of this system considering health reform trends and initiatives.

\section{The Methodology of the Study}

This research is conducted through an anonymous annual survey of responsible persons of the decision-making hierarchy in the public health institutions such as: tertiary health institutions, reffering to university clinics, secondary health institutions, reffering to clinical hospitals, general hospitals, special hospitals and entities of the Republic of Macedonia. The research was conducted over a period of one year, from August 2017 to August 2018, regarding the most important issues faced by the managers of health institutions and their impact on the current health system and their expectations with plans regarding future projections.

The findings and results of this research reflect the responses of 80 surveys successfully completed, with the competent persons of institutional management staff, from a total of 100 questionnaires sent to health institutions of different levels in the Republic of Macedonia. For this research survey questionnaires were used, 
consisting of standardized questions, focused mainly on how the managers evaluate and prepare for the future health system, which will continue to be dynamic and complex. The respondents' responses to this issue were anonymous. This study is based on thorough and careful processing of survey data and content analysis of responses given using standardized approaches to grouping them.

\section{Results}

From this annual survey conducted through survey questionnaires among the top managers of the public health institutions, the following results are obtained about the predictions of directors of health institutions for the future of healthcare system:

The new model of value-based healthcare will reformulate and change the future of healthcare. Health services in hospitals will be paid in different ways, making profitability more difficult. Most of the services will be ambulatory or home-based. Leaders or executives expect this value-based model to set in motion further consolidation, purchases, acquisitions, mergers between hospitals and doctors because it has the potential to improve access to capital, develop the monetary market, and increase the skills and opportunities that are believed to be necessary in order to be successful in contracting of the value-based healthcare.

According to the interviewed leaders, the Value-Based Healthcare Model is the most important trend of hospitals' confrontation for the next five years. Respondents stressed out that the Value-Based Health Care will submerge the traditional healthcare model because they are aware that patients are increasingly demanding fast, quality and efficient health services and health care. As a result of which health institutions will have to respond by reducing stationary use and achieving better performance and results.

Orientation towards patients and the need to be more friendly, direct and closer to patients are part of the future. Hospital staff will need to be more accessible and transparent, and also this staff will have to provide quality services and such interactions at the time and place that the patients require. More than half of the surveyed leaders agree that the healthcare system is transforming into a patientcentered model. Patients are becoming more selective, sharp and shrewd about their medical treatment; they have greater financial responsibility and require that medical care be more convenient and accessible.

Some of the leaders emphasize that with the new patient-centered model, the medical care offered at the clinics by the work teams will be safer, less costly and more appropriate for the patients. To achieve these goals, hospitals need to review and revaluate their existing medical care models, change their organizational culture, and invest in new technologies needed for the implementation of a new model of medical care.

The technological advancement that, according to most leaders, is the one that will significantly improve future healthcare by making the services more accessible, 
information more transparent as well as prepare the doctors and other medical staff to be able to provide a better healthcare. Especially in the next decade, around 2028, it is thought that open technology will strongly support electronic visits and will allow providers and patients to exchange relevant and rapid data and information. In addition, it is highly likely that health institutions and medical staff will use multiple analytical processes to achieve better patient health care, better patient health management, and achieve better results.

Respondents also predict that large volumes of healthcare data will support quality findings, cost effectiveness, and medical research through knowledge derived from predictive modeling with extended medical and other data. This modeling could include identifying the high-risk population and forecasting expenditure or outcomes for the specific part of the population - patients. Technological investments will also support the health system's ability to take on the financial risk for the population.

Separate data review includes the promise of cost reduction through identifying efficient healthcare structures and models and high-risk population. Most respondents emphasized that investing in technological risk management capabilities related to patient management is needed from the perspective of spending. The challenges in the realization of this vision are: numerous and different data from which appropriate decisions should be made; incomplete data interrelated between health systems and doctors; lack of skills for predictive models and experience.

The talent - respondents agree that the discovery and development of the talent are essential elements for achieving success in the labor market. All respondents highlighted the long-term priorities of the talents:

Doctors / Clinical Leaders who treat and respect other doctors and general medical staff of their institution as true colleagues and as the most valuable asset possible. Leaders who through behavior and correct actions are able to motivate employees and influence them for the successful realization of the organizational goals.

Leaders who can apply knowledge and experience from other areas to bring innovations to health care. Among the most wanted are the leaders who want to undertake moderate risks and change the status quo.

Computer specialists with analytical skills who have the ability to exchange data from different sources and technologies into useful information and knowledge. Particularly, individuals with knowledge of technical and social sciences are also needed to help manage the healthcare of the population through predictive models.

\section{Respondents repeatedly emphasized the need for:}

The leaders to be:

Inovative 
Visionary

Entrepreneurs willing to take moderate risks

Accepting of new models for health care

Focused on the patient

\section{These leaders also stressed the need for the employees to be:}

Able to follow the changes

Ready to work in groups

Engaging and participating in the work processes, motivated and enthusiastic.

\section{Conclusion}

Regarding the sources of considerable concerns presented to the directors of healthcare institutions, can be emphasized that healthcare managers feel great uncertainty about the dynamic and complex environment they are operating in, the overall economic situation and the possibility of increasing income. They are focused mainly on the implementation of contemporary technology for creating new values in new forms, developing new and dynamic partnerships and reducing the spendings.

If they carry out good healthcare planning, they manage to attract and retain quality and talented human resources, and build well-thought out and thoughtful strategies; healthcare providers will face multiple shocks of the dynamic and complex environment and will face, manage and efficiently carry out their healthcare activities in the next very challenging decade.

In today's healthcare system there is a lack of innovators and those with high readiness to undertake moderate risk that will be greatly needed in the future.

Therefore, we can say that:

Physician and visionary leaders, even though are scarce nowadays, are much needed.

Some of the interviewed leaders point out that the hospitals have noticed the lack of information technology specialists and those with analytical skills.

Others mentioned that also successful planning is a very important issue. They require the team of leaders to identify clear organizational objectives and goals, to draft plans and strategies and to design adecuate programs and policies for their realization in order to survive, develop, and adapt the institution to the dynamic changes in the dynamic and complex environment.

One of the important objectives of health care providers is to focus their efforts on the development and support of talented staff in order to create the necessary staff to achieve success in the future, which is thought to be very challenging. 
Health institutions should also invest in new strategies for motivating, promoting positive employee behavior and raising their behavior awareness in the dynamic, complex environment.

The priorities needed to achieve high performance in the next years are:

Continuous and stable reduction of costs - always by continuing to respond to the phenomenon of reduction of operational boundaries.

Defining an adequate method of how to approach the consolidation and the change in the area of services provided - to gain spending efficiency and provide quality medical care. Huge institutions can exist, each networked or consolidated, if leaders develop their strategies according to the circumstances.

Bringing the decisions that the right services are provided in the right place - the goal is to minimize the phenomenon of providing dual services in different places and concentrate and provide them in places where the cost for their delivery is lower.

The new approach of careful treatment of patients has already begun to change medicine and it is predicted that this development trend will prevail even in next years. The specific changes include:

The patient in the first place - it may happen that within 24 hours the patients asks for a visit and a check up, doctors will need to provide healthcare also during the weekends and the actions taken for such care will be more appropriate even when they are offered at home.

Digital technologies will improve access and will create new opportunities for patients to meet and consult doctors - video and electronic visits will increase, reducing traditional personal visits to doctors' offices or hospitals.

Practices for quality health care will increase in the way that this will be engaged and cared for by other medical staff - internship nurses and other practitians will provide the widest range of services. This category of employees can reduce costs for patients and be in the most suitable places.

One of the biggest challenges for a successful patient-oriented model is to change organizational culture and staff attitudes. To motivate medical staff to be open to change, a powerful authoritative leadership is needed. Industries such as gastronomy, catering, and the various cosmetic services markets have been the source of leading practices in how to connect the market and customers in healthcare.

Therefore, we can conclude that institutional development requires:

Forecasting and precise planning of actions for the future, which is half of the success in this future. Although the leaders of the health institutions have a clear vision and understanding for the future, different obstacles may arise, therefore there should be a set of operational, tactical and strategic objectives and goals on an institutional level. 
In strategic terms, the main issue is related to the type of investment that should be made at the moment in order to gain the highest competitive advantage in the future and the decision to be taken for the best positioning of the institution to achieve high success during the next five years but always focusing on maximizing the needs and requirements of patients while being rational in delivering quality health services.

The advanced talent and technology are the top priorities and the development of each one of them can undoubtedly require great investment, effort and expense. It is quite challenging and complex that the directors need to follow and develop these investments on a regular basis to better position their institution towards a quality healthcare model - value-based healthare and an operating environment in which meeting the needs and requirements of the patient is a key issue.

The path to success is different for all market and health institution, so the leaders, along with their leading teams, need to continue with the models of value-based healthcare in the institutions they manage; to improve the quality of the services provided to patients; to strive and seek appropriate purchases or partnerships when they are needed; to develop new technological and analytical skills and learn from the successes of others both inside and outside the health sphere.

\section{References}

[1] Flunn W, Mathis R, Jackson J, Langan P. Healthcare Human Resource Management Mason, OH: Thomas South - Western; 2004.

[2] Law on Healthcare in the Republic of Macedonia.

[3] Law on public healthcare in the Republic of Macedonia.

[4] Law on the protection of rights of patients in the Republic of Macedonia.

[5] Leiyu Shi, Jones and Bartlett Publishers, Managing human resources in healthcare organizations, Inc., Ontario, Canada, 2007;157-185.

[6] Nadler David, Shaw Robert, Walton A. Elise and associates. Organizational architecture: Designing for high performance. "Discontinuous change: Leading Organizational Trannsformations". San Francisco: Jossey - Bass Inc, 1995.

[7] Reforms in the Healthcare System in the Republic of Macedonia www.crpm.org.mk

[8] Robey Daniel. Designing organizations, Third edition IRWIN, Burr Ridge, Illinois, 1991.

[9] Sherman Folland, Allen Goodman, Miron Stano. Economics of Health and Health Care, 2008.

[10] Termino, E \& Webster, E.G., Primary Health Concepts and challenges in a changing world: Altna - Ata revisited. Geneva, World Health Organization. 1997.

[11] World Health Organization, Report on healthcare in transition, 2000

[12] Zeqiri I., Menaxhmenti, Tetovë 2006. 